\title{
Efficacy and Safety of All-oral Emitasvir and Sofosbuvir in Patients with Genotype 1b HCV Infections without Cirrhosis
}

\author{
Huiying Rao ${ }^{1}$, Xingxiang Yang ${ }^{2}$, Youwen $\operatorname{Tan}^{3}$, Qin Ning ${ }^{4}$, Daokun Yang ${ }^{5}$, Jiefei Wang ${ }^{6}$ \\ Yongfeng Yang ${ }^{7}$, Sujun Zheng ${ }^{8}$, Dongliang Yang ${ }^{9}$, Jinlin Hou $^{10}$, Qing Xie ${ }^{11}$, Caiyan Zhao ${ }^{12}$, \\ Lunli Zhang ${ }^{13}$, Xiaorong Mao ${ }^{14}$, Tong Sun ${ }^{15}$, Lang Bai ${ }^{16}$, Fuchun Zhang ${ }^{17}$, Jinglan Jin ${ }^{18}$, \\ Yingren Zhao ${ }^{19}$, Maorong Wang'20, Wen Xie ${ }^{21}$, Yingjie Ma22, Jun Quan ${ }^{23}$, Xuebing Yan ${ }^{24}$, \\ Ping $\mathrm{An}^{25}$, Feng $\mathrm{Lin}^{26}$, Jidong Jia ${ }^{27}$, Xiaoxuan $\mathrm{Hu}^{28}$, Zuojiong Gong ${ }^{29}$, Jie $\mathrm{Wu}^{30}$, \\ Yongping Chen ${ }^{31}$, Zhansheng Jia ${ }^{32}$, Minghua Lin $^{33}$, Guiqiang Wang ${ }^{34}$, Yueyong Zhu ${ }^{35}$, \\ Yingjun Zhang*36, Hongming Xie ${ }^{36}$, Lin Luo ${ }^{36}$, Qingyun Ren ${ }^{36}$, Rui Huang ${ }^{1}$ and Lai Wei*37
}

\begin{abstract}
${ }^{1}$ Peking University People's Hospital, Peking University Hepatology Institute, National Clinical Research Center for Infectious Disease, Beijing Key Laboratory of Hepatitis C and Immunotherapy for Liver Diseases, Beijing International Cooperation Base for Science and Technology on NAFLD Diagnosis, Beijing, China; ${ }^{2}$ Department of Infectious Diseases, Sichuan Provincial People's Hospital, Chengdu, Sichuan, China; ${ }^{3}$ Department of Hepatology, The Third People's Hospital of Zhenjiang, Zhenjiang, Jiangsu, China; ${ }^{4}$ Department of Infectious Diseases, Tongji Hospital, Tongji Medical College Huazhong University of Science and Technology, Wuhan, Hubei, China; ${ }^{5}$ Department of Infectious Diseases, The First Affiliated Hospital of Xinxiang Medical College, Xinxiang, Henan, China; ${ }^{6}$ Shanghai Public Health Clinical Center, Shanghai, China; ${ }^{7}$ Department of Hepatology, The Second Hospital of Nanjing, Nanjing, Jiangsu, China; ${ }^{8}$ Center of Artificial liver, Beijing Youan Hospital, Capital Medical University, Beijing, China; ${ }^{9}$ Department of Infectious Disease, Union Hospital, Tongji Medical College, Huazhong University of Science and Technology, Wuhan, Hubei, China; ${ }^{10}$ Department of Infectious Diseases, Nanfang Hospital, Southern Medical University, Guangzhou, Guangdong, China; ${ }^{11}$ Department of Infectious Diseases, Ruijin Hospital Affiliated to Shanghai Jiaotong University School of Medicine, Shanghai, China; ${ }^{12}$ Department of Infectious Diseases, The Third Hospital of Hebei Medical University, Shijiazhuang, Hebei, China; ${ }^{13}$ Department of Infectious Diseases, The First Affiliated Hospital of Nanchang University, Nanchang, Jiangxi, China; ${ }^{14}$ Department of Infectious Diseases, The First Hospital of Lanzhou University, Lanzhou, Gansu, China; ${ }^{15}$ Department of Infectious Diseases, Wuxi Fifth People's Hospital, Wuxi, Jiangsu, China; ${ }^{16}$ Infectious Diseases Center, West China Hospital, Sichuan University, Chengdu, Sichuan, China; ${ }^{17}$ Department of Hepatology, Guangzhou Eighth People's Hospital, Guangzhou, Guangdong, China; ${ }^{18}$ Department of Hepato-Biliary-Pancreatic Diseases, The First Hospital of Jilin University, Changchun, Jilin, China; ${ }^{19}$ Department of Infectious Diseases, Xi'an Jiaotong University First Affiliated Hospital, Xi'an, Shaanxi, China; ${ }^{20}$ Department of Infectious Diseases, DiBaYi Hospital of the Chinese People's Liberation Army, Nanjing, Jiangsu, China; ${ }^{21}$ Hepatology Center, Beijing Ditan Hospital, Capital Medical University, Beijing, China; ${ }^{22}$ Department of Infectious Diseases, Zhengzhou People's Hospital, Zhengzhou, Henan, China; ${ }^{23}$ Department of Infectious Diseases, Xiangya Hospital, Central South University, Changsha, Hunan, China; ${ }^{24}$ Department of Infectious Diseases, Xuzhou Medical College Affiliated Hospital, Xuzhou, Jiangsu, China; ${ }^{25}$ Department of Infectious Diseases, Shenyang Sixth People's Hospital, Shenyang, Liaoning, China;

${ }^{26}$ Department of Infectious Diseases, Hainan General Hospital, Haikou, Hainan, China; ${ }^{27}$ Hepatology Center, Beijing Friendship Hospital, Capital Medical University, Beijing, China; ${ }^{28}$ Department of Infectious Diseases, Hunan Provincial People's Hospital, Changsha, Hunan, China; ${ }^{29}$ Department of Infectious Diseases, Renmin Hospital of Wuhan University, Wuhan, Hubei, China; ${ }^{30}$ Department of Infectious Diseases, Wuhan central hospital, Wuhan, Hubei, China; ${ }^{31}$ Department of Infectious Diseases, The First Affiliated Hospital of Wenzhou Medical University, Wenzhou, Zhejiang, China; ${ }^{32}$ Department of Infectious Diseases, Tangdu Hospital, The Fourth Military Medical University of the People's Liberation Army, Xi'an, Shaanxi, China; ${ }^{33}$ Department of Hepatology, Meng Chao Hepatobiliary Hospital, Fujian Medical University, Fuzhou, Fujian, China; ${ }^{34}$ Department of Infectious Diseases, Peking University First Hospital, Beijing, China; ${ }^{35}$ Department of Infectious Diseases, The First Affiliated Hospital of Fujian Medical University, Fuzhou, Fujian, China; ${ }^{36}$ State Key Laboratory of Anti-Infective Drug Development
\end{abstract} (NO.2015DQ780357), Sunshine Lake Pharma Co., Ltd, Dongguan, Guangdong, China; ${ }^{37}$ Hepatopancreatobiliary Center, Beijing Tsinghua Changgung Hospital, Tsinghua University, Beijing, China

\footnotetext{
Keywords: Hepatitis C virus; Genotype 1; Direct acting antivirals; Emitasvir; Sofosbuvir; Combination treatment.

Abbreviations: AEs, adverse events; ALT, alanine aminotransferase; AST, aspartate aminotransferase; BMI, body mass index; CI, confidence interval; DAA, direct-acting antiviral; HCV, hepatitis C virus; LLOQ, lower limit of quantitation; LSM, liver stiffness modulus; NS5A, nonstructural protein 5A; RASs, resistance-associated substitutions; SAES, serious AES; SD, standard deviation; SVR, sustained virologic response; ULN, upper limit of normal.

Received: 13 April 2020; Revised: 5 July 2020; Accepted: 12 August 2020

*Correspondence to: Lai Wei, Hepatopancreatobiliary Center, Beijing Tsinghua Changgung Hospital, Tsinghua University, No. 168, Litang Road, Changping District, Beijing 102218, China. Tel: +86-10-56118881, Fax: +86-10-56118500, E-mail: weilai@mail.tsinghua.edu.cn; Yingjun Zhang, State Key Laboratory of Anti-Infective Drug Development (NO. 2015DQ780357), Sunshine Lake Pharma Co., Ltd., No. 368, Zhen'an Road, Chang'an Town, Dongguan, Guangdong 523871, China. Tel: +86-769-886158882337, Fax: +86-769-85370222-2337, E-mail: zhangyingjun@hec.cn
} 


\section{Abstract}

Background and Aims: Emitasvir is a new type of hepatitis $\mathrm{C}$ virus (HCV) nonstructural protein 5A (NS5A) inhibitor, and the data of phase 2 trial has shown emitasvir-sofosbuvir to have good safety and tolerance. We conducted this phase 3 trial to further verify the efficacy and safety. Methods: We evaluated the antiviral activity and safety of a 12-week regimen of emitasvir phosphate $(100 \mathrm{mg})$ combined with sofosbuvir $(400 \mathrm{mg}$ ) once daily in non-cirrhotic patients with genotype $1 \mathrm{HCV}$ infection. The primary endpoint was a sustained virological response at 12 weeks (SVR12) after the end of treatment. Results: Of the 362 patients enrolled in the trial, $39.8 \%$ were male, $99.2 \%$ had HCV genotype $1 \mathrm{~b}, 0.8 \%$ had genotype $1 \mathrm{a}$ and $79.8 \%$ were treatment-naïve. The average age was 47.2 years. All patients completed the treatment and follow-up. All 3 patients with genotype 1 a achieved SVR. Two genotype $1 \mathrm{~b}$ treatment-naïve patients experienced virologic relapse. The rate of SVR12 was $99.7 \%$ (358/359), and SVR24 was 99.4\% (357/359) in genotype 1b. Overall, 36.2\% had resistance-associated substitutions (RASs) in NS5A and $98.3 \%$ had RASs in NS5B at baseline. The RASs at baseline had no effect on the rates of response. Serious adverse events were reported in 16 patients and were not related to emitasvir-sofosbuvir. Most adverse events did not require therapy. Conclusions: The 12 weeks of treatment with emitasvir-sofosbuvir was a highly efficient and safe treatment for a wide range of patients with HCV genotype $1 \mathrm{~b}$ infection without cirrhosis, who had not been treated or who had been treated with interferon-based regimen previously.

Citation of this article: Rao $H$, Yang $X$, Tan $Y$, Ning $Q$, Yang D, Wang J, et al. Efficacy and safety of all-oral emitasvir and sofosbuvir in patients with genotype $1 \mathrm{~b} \mathrm{HCV}$ infections without cirrhosis. J Clin Transl Hepatol 2020;8(3):255-261. doi: 10.14218/JCTH.2020.00031.

\section{Introduction}

Hepatitis $C$ is a global epidemic, and people of different sex age and race are susceptible to infection with the hepatitis $C$ virus (HCV). The World Health Organization estimates that 71 million people worldwide had chronic HCV infection in 2015 and that 399,000 people died of liver cirrhosis or hepatocellular carcinoma caused by HCV infection in $2015 .^{1}$ In 2006, the positive rate for anti-HCV antibodies was $0.43 \%$ in the Chinese population of individuals ages 1-59 years-old. ${ }^{2}$ From this, it was estimated that there are 5.6 million people infected with HCV in the general population in China. However, when including the high-risk groups and high-risk areas of HCV infection, China is expected to have a total of about 10 million cases of HCV infection. ${ }^{2}$

In China, genotypes $1 \mathrm{~b}$ and $2 \mathrm{a}$ are common, with the former being the most common, accounting for $56.8 \%$, while genotype $1 \mathrm{a}$ accounts for only $1.4 \%$. Genotypes 4 and 5 are very rare, and type 6 is relatively rare..$^{3,4}$ The sustained virologic response (SVR) rate of pegylated-interferon combined with ribavirin in HCV genotype 1 patients without cirrhosis is reportedly about $75 \% .^{5}$ The application of all-oral directacting antiviral (DAA) therapy in China starting in 2017 has led to improvements in both treatment options and outcomes for patients with HCV infection, and the SVR rate has reached more than $95 \% .^{6-9}$ Patients with genotype 1 can be treated with either pangenotypic DAAs or genotype-specific DAAs.
Emitasvir phosphate is a new type of HCV nonstructural protein 5A (NS5A) inhibitor independently developed in China. Preclinical studies showed that the compound has strong antiviral activity against genotype $1 \mathrm{HCV}$. The data of phase 1 and phase 2 clinical trials showed that the combination regimen of emitasvir-sofosbuvir has good safety and tolerance. ${ }^{10}$ In the phase 3 clinical trial described herein, we evaluated the antiviral activity and safety of a 12-week regimen of emitasvir phosphate combined with sofosbuvir in non-cirrhotic patients with genotype $1 \mathrm{HCV}$ infection, who were treatment-naïve (defined as patients who had never been treated for their HCV infection) or treatment-experienced (defined as patients who were previously treated with an interferon-based regimen).

\section{Methods}

\section{Study design and treatment}

This phase 3, single arm, open-label, multicenter study involved treatment-naïve or treatment-experienced, noncirrhotic patients with chronic HCV genotype 1 infections, and was conducted at 36 study sites in mainland China between April 2018 and June 2019 (funded by Sunshine Lake Pharma Co., Ltd.; ClinicalTrials.gov number, NCT 03487107). All patients were treated by $100 \mathrm{mg}$ emitasvir capsule (Yichang HEC Changjiang Pharmaceutical Co., Ltd, China) plus $400 \mathrm{mg}$ sofosbuvir tablet (Gilead, USA), and administered orally once daily. The duration of treatment was a consecutive 12 weeks, after which all study participants entered a 24-week treatment-free follow-up period.

This clinical trial was approved by each participating center's independent ethics review board and conducted in accordance with the principles of the Declaration of Helsinki, good clinical practice, and Chinese legal and regulatory requirements. Prior to the beginning of the trial, the subject's written informed consent was obtained.

\section{Patients}

The study population included male and female patients aged over 18 years old, body mass index (BMI) between 18 and 32 $\mathrm{kg} / \mathrm{m}^{2}$, and body weight $\geq 40 \mathrm{~kg}$. For study participation, all female patients had to indicate they had no plans to become pregnant during the screening period, and from the beginning of treatment to 90 days after treatment, the patients had to agree to use of effective contraception. The patients had chronic HCV infection of genotype 1 without cirrhosis, and had an HCV RNA level $\geq 10,000 \mathrm{IU} / \mathrm{mL}$ at the time of screening. The treatment-naïve patients were defined as having no prior exposure to any interferon, ribavirin, or other approved or experimental DAAs. The treatment-experienced patients were defined as having received interferon-based regimen therapy, with completion being at least 2 months before the screening visit. Patients had FibroScan ${ }^{\circledR}$ liver stiffness modulus (LSM) that did not exceed $12.5 \mathrm{kPa}$ at 6 months prior to enrollment or had no cirrhosis confirmed by liver biopsy at 24 months prior to enrollment. Liver biopsy results were preferred to FibroScan ${ }^{\circledR}$ LSM.

Key exclusion criteria included the following: clinical hepatic decompensation or a history of hepatic decompensation, such as ascites, hepatic encephalopathy, or esophageal or gastric varices bleeding; the existence of primary liver diseases not caused by $\mathrm{HCV}$; receipt of any DAAs before 
screening; cardiovascular events that had occurred in the 6 months prior to screening; gastrointestinal diseases or postoperative status affecting drug absorption; history of mental illness or related diseases; malignancy within 5 years prior to signing of the informed consent; solid organ transplantation; hepatitis $B$ virus surface antigen or anti-human immunodeficiency virus serological test result positivity; confirmed primary liver cancer; alpha-fetoprotein $>100 \mathrm{ng} / \mathrm{mL}$ (if 20 $\mathrm{ng} / \mathrm{mL} \leq$ alpha-fetoprotein $\leq 100 \mathrm{ng} / \mathrm{mL}$, ultrasound liver examination was required to exclude primary hepatocellular carcinoma); serum alanine aminotransferase (ALT) or aspartate aminotransferase (AST) $>10$ upper limit of normal; total bilirubin $>1.5$ upper limit of normal; albumin $<3.5 \mathrm{~g} / \mathrm{dL}$; international normalized ratio $>1.5$; absolute neutrophil count $<1.5 \times 10^{9} / \mathrm{L}$; platelet count $<90 \times 10^{9} / \mathrm{L}$; hemoglobin $<110 \mathrm{~g} / \mathrm{L}$ (for females) or $120 \mathrm{~g} / \mathrm{L}$ (for males); HbA1c $>8.5 \%$; creatinine clearance rate $<50 \mathrm{~mL} / \mathrm{min}$, as calculated by the Cockcroft-Gault formula; in the absence of effective control, any heart, respiratory, gastrointestinal tract, blood, nerve or other diseases, or diseases whose treatment may interfere with the participants ability to obey the research plan or assessment of the study investigators; a woman or man whose partner was pregnant; and any other conditions the investigator considered inappropriate for exclusion.

\section{Study assessments}

HCV RNA was determined using the COBAS AmpliPrep/ COBAS Taqman HCV Test Version 2.0 Virus Quantitative Detection Kit (Roche Molecular System, Inc., USA), with a lower limit of quantification (LLOQ) of $15 \mathrm{IU} / \mathrm{mL}$. HCV genotyping was directly sequenced by reverse transcription PCR (sensitivity $20 \%$ ). HCV RNA and genotyping were evaluated in the clinical laboratory of Guangzhou Kingmed Center (China). Other clinical laboratory assessments were mainly conducted in local hospital laboratories. Blood samples were collected to determine serum levels of HCV RNA before the initiation of emitasvir- sofosbuvir and at weeks $1,2,4,6,8$,
10 and 12 during treatment and at weeks 4, 12 and 24 after treatment.

Resistance testing was conducted in the clinical laboratory of Guangzhou Kingmed Center, using Sanger sequencing of the NS5A and NS5B regions (threshold $\geq 20 \%$ of the viral population) in samples of all patients at baseline and at the time of treatment failure. The amino acid sites at 24, 28, 30, 31, 32, 58, 92 and 93 of HCV NS5A, and 61, 62, 159, 202, $282,316,320,321$ and 440 of HCV NS5B were analyzed based on Sanger sequencing results.

Adverse events (AEs), mainly those occurring during treatment, as well as physical examination, vital signs, and findings from 12-lead electrocardiogram, laboratory examination, and abdominal ultrasound were included in safety assessments. AEs were coded using the MedDRA 22.0 dictionary.

\section{Study endpoints}

The primary outcome endpoint was the percentage of subjects who achieved HCV RNA <LLOQ or HCV RNA at undetectable level at 12 weeks after the end of treatment (SVR12). The secondary outcome endpoints were the percentage of subjects who achieved HCV RNA <LLOQ or HCV RNA at undetectable level at 4 and 24 weeks after the end of treatment (SVR4 and SVR24), the percentage of subjects with virological failure during treatment or relapse after treatment, and HCV virus resistance monitored at baseline, during and after treatment.

Safety endpoints included AEs during treatment and serious AEs (SAEs), vital signs, findings from physical examinations, 12-lead electrocardiogram, laboratory tests and abdominal ultrasound, and other safety indicators.

\section{Statistical analyses}

Statistical analysis of data was performed using the statistical analysis software SAS Version 9.4 (SAS Institute Inc., USA). Continuous data were analyzed using descriptive statistical

Table 1. Baseline demographics and disease characteristics

\begin{tabular}{|c|c|c|c|}
\hline Characteristic & Genotype $1 a, n=3$ & Genotype $1 b, n=359$ & Overall, $n=362$ \\
\hline Mean age (range), years & $35.7(24-46)$ & $47.3(20-78)$ & $47.2(20-78)$ \\
\hline Age $\geq 65$ years, $n(\%)$ & 0 & $31(8.6)$ & $31(8.6)$ \\
\hline Male, $n(\%)$ & $2(66.7)$ & $142(39.6)$ & $144(39.8)$ \\
\hline Mean BMI (range), kg/m ${ }^{2}$ & $23.10(18.2-27.7)$ & $23.44(18.0-32.0)$ & $23.44(18.0-32.0)$ \\
\hline \multicolumn{4}{|l|}{ Prior treatment history } \\
\hline Treatment-naïve, $n(\%)$ & $2(66.7)$ & 287 (79.9) & $289(79.8)$ \\
\hline Treatment-experienced, $n(\%)$ & $1(33.3)$ & $72(20.1)$ & $73(20.2)$ \\
\hline FibroScan ${ }^{\circledR}$ LSM, mean (SD), kPa & $6.23(2.627)$ & $6.81(2.619)$ & $6.80(2.616)$ \\
\hline \multicolumn{4}{|l|}{ HCV RNA } \\
\hline Mean (SD), Log10 IU/mL & $6.43(0.404)$ & $6.19(0.781)$ & $6.20(0.779)$ \\
\hline$\geq 800,000 \mathrm{IU} / \mathrm{mL}, n(\%)$ & $3(100.0)$ & $261(72.7)$ & $264(72.9)$ \\
\hline \multicolumn{4}{|l|}{ ALT } \\
\hline Mean (SD), IU/L & $50.93(29.029)$ & $63.04(54.341)$ & $62.94(54.169)$ \\
\hline$>1.5$ ULN, $n(\%)$ & 0 & $117(32.6)$ & $117(32.3)$ \\
\hline
\end{tabular}

Abbreviations: ALT, alanine aminotransferase; BMI, body mass index; LSM, liver stiffness modulus; SD, standard deviation; ULN, upper limit of normal. 
Rao H. et al: Emitasvir and sofosbuvir in HCV genotype $1 \mathrm{~b}$

methods, such as case number, mean, standard deviation, minimum, and maximum. The frequency and classification data were analyzed using frequency and percentage. All subjects enrolled and taking at least one dose of the study drug were included in the full analysis set. The SVR12 point estimate and the bilateral 95\% confidence interval (CI) were based on the full analysis set. All enrolled subjects taking at least one dose of the study drug were included in the safety set. All security analysis was based on safety set.

\section{Results}

\section{Demographics and baseline characteristics}

Of the 440 screened patients, 362 were enrolled. Among them, 3 patients had HCV genotype $1 \mathrm{a}$ and 359 patients had HCV genotype 1b. All 362 subjects completed the 12-week treatment period. One treatment-naïve patient with genotype $1 \mathrm{~b}$ experienced a virologic relapse during the follow-up at 12 weeks after treatment. The other 361 patients completed the follow-up 24 weeks after treatment. As shown in Table 1, the mean age of the patients was 47.2 years, mean weight was $62.26 \mathrm{~kg}$, and mean BMI was $23.44 \mathrm{~kg} / \mathrm{m}^{2}$. Among the patients, $144(39.8 \%)$ were male and $218(60.2 \%)$ were female, $353(97.5 \%)$ were Han nationality and $9(2.5 \%)$ were other nationalities.

Of 362 subjects, 289 (79.8\%) were treatment-naïve, including 2 cases of genotype $1 \mathrm{a}$ and 287 cases of genotype 1b. There were $73(20.2 \%)$ who were treatment-experienced, including 1 of genotype $1 a$ and 72 cases of genotype 1b. Mean baseline HCV RNA was $6.20( \pm 0.779) \mathrm{Log} 10 \mathrm{IU} / \mathrm{mL}$, and 264 patients $(72.9 \%$ ) had a baseline HCV RNA $\geq 800,000$ $\mathrm{IU} / \mathrm{mL}$. Mean baseline ALT was $62.94( \pm 54.169) \mathrm{U} / \mathrm{L}$, and mean baseline FibroScan ${ }^{\circledR}$ LSM was $6.80( \pm 2.616) \mathrm{kPa}$.

\section{Baseline resistance-associated substitutions (RASs)}

As shown in Table 2, among the 3 subjects with genotype 1 , $1(33.3 \%)$ had M28V mutation in NS5A at baseline. Three $(100 \%)$ subjects had RASs in NS5B at baseline, of which two $(66.7 \%)$ had mutations in S62N and one $(33.3 \%)$ had mutation in S62D. Among the 359 subjects with genotype $1 \mathrm{~b}, 130(36.2 \%)$ subjects had RASs in NS5A at baseline. The mutation rates of $\mathrm{R} 30 \mathrm{Q}, \mathrm{Y} 93 \mathrm{Y} / \mathrm{H}$ and $\mathrm{Y} 93 \mathrm{H}$ were more than $5 \%$, and were $11.1 \%(40 / 359), 8.4 \%$ (30/359) and $5.6 \%(20 / 359)$ respectively with no P32 site mutation. A total of $353(98.3 \%)$ subjects had RASs in NS5B at baseline. The mutation rates of $\mathrm{C} 316 \mathrm{~N}, \mathrm{E} 440 \mathrm{E} / \mathrm{G}$ and $\mathrm{E} 440 \mathrm{G}$ were $97.5 \%$ (350/359), $5.8 \%(21 / 359)$ and $5.6 \%(20 / 359)$ respectively. No mutations were found at D61, S282, L320 and V321 sites.

\section{Efficacy}

All subjects had good adherence to drug exposure time and dose, ranging from $80-120 \%$. All 362 subjects achieved HCV RNA <LLOQ at 4 weeks after treatment. Except for 1 treatment-naïve patient with genotype $1 \mathrm{~b}$ who was confirmed to have a virological relapse at 12 weeks after treatment; the other 361 subjects all had lower HCV RNA than LLOQ at 12 weeks after treatment, with SVR12 reaching $99.7 \%$ (95\%CI: $98.47-99.99 \%)$. Another genotype $1 \mathrm{~b}$ treatment-naïve patient developed virological relapse at 24 weeks after treatment, with SVR24 at 99.4\% (360/362). Both SVR4, SVR12
Table 2. Baseline resistance-associated substitutions in genotype 1

\begin{tabular}{|c|c|c|}
\hline & $\begin{array}{l}\text { Genotype } 1 \mathrm{a}, \\
n=3\end{array}$ & $\begin{array}{l}\text { Genotype } 1 \mathrm{~b} \\
n=359\end{array}$ \\
\hline \multicolumn{3}{|l|}{ HCV NS5A } \\
\hline RASs & $1(33.3)$ & $130(36.2)$ \\
\hline R30Q & & $40(11.1)$ \\
\hline $\mathrm{Y93Y/H}$ & & $30(8.4)$ \\
\hline Y93H & & $20(5.6)$ \\
\hline P58S & & 14 (3.9) \\
\hline R30Q/R & & $12(3.3)$ \\
\hline L28M & & $5(1.4)$ \\
\hline $\mathrm{M} 28 \mathrm{~V}$ & $1(33.3)$ & \\
\hline No RASs & $2(66.7)$ & $229(63.8)$ \\
\hline \multicolumn{3}{|l|}{ HCV NS5B } \\
\hline RASs & $3(100)$ & $353(98.3)$ \\
\hline C316N & & $350(97.5)$ \\
\hline $\mathrm{E} 440 \mathrm{E} / \mathrm{G}$ & & $21(5.8)$ \\
\hline E440G & & $20(5.6)$ \\
\hline $\begin{array}{l}\text { C316N, } \\
\text { E440G }\end{array}$ & & $18(5.0)$ \\
\hline $\begin{array}{l}\text { C316N, } \\
\text { E440E/G }\end{array}$ & & $17(4.7)$ \\
\hline D62N & & $14(3.9)$ \\
\hline D62D/N & & $13(3.6)$ \\
\hline $\mathrm{D} 62 \mathrm{~N}, \mathrm{C} 316 \mathrm{~N}$ & & $13(3.6)$ \\
\hline $\begin{array}{l}\text { D62D/N, } \\
\text { C316N }\end{array}$ & & $10(2.8)$ \\
\hline E202D & & $5(1.4)$ \\
\hline L159F & & $5(1.4)$ \\
\hline $\begin{array}{l}\text { E202D, } \\
\text { C316N }\end{array}$ & & $5(1.4)$ \\
\hline $\begin{array}{l}\text { L159F, } \\
\text { C316N }\end{array}$ & & $5(1.4)$ \\
\hline $\mathrm{S} 62 \mathrm{~N}$ & $2(66.7)$ & \\
\hline S62D & $1(33.3)$ & \\
\hline No RASs & 0 & $6(1.7)$ \\
\hline
\end{tabular}

Data are presented as $n(\%)$

Abbreviations: HCV, hepatitis C virus; NS5A, nonstructural protein 5A; NS5B, nonstructural protein $5 B$; RASs, resistance-associated substitutions.

and SVR24 of HCV genotype 1a subjects were $100.0 \%(3 / 3)$, while SVR4, SVR12 and SVR24 of HCV genotype $1 \mathrm{~b}$ subjects were $100.0 \%$ (359/359), $99.7 \%$ (358/359), and $99.4 \%$ (357/359) respectively.

In this study, very high SVR12 was obtained in all subgroups (Table 3), including treatment-naïve vs. treatment-experienced, $<65$ years vs. $\geq 65$ years, and male vs. female, as well as subdivisions based on baseline BMI level, baseline HCV RNA level and baseline ALT level. For example, the SVR12 of the treatment-naïve and treatment-experienced subjects was $99.7 \%$ (288/289) and $100.0 \%(73 / 73)$ respectively. The efficacy of each subgroup is shown in 
Rao H. et al: Emitasvir and sofosbuvir in HCV genotype $1 \mathrm{~b}$

Table 3. Efficacy of treatment by subgroup

\begin{tabular}{|c|c|c|c|c|c|c|}
\hline & \multicolumn{2}{|c|}{ Genotype $1 a, n=3$} & \multicolumn{2}{|c|}{ Genotype $1 \mathrm{~b}, n=359$} & \multicolumn{2}{|c|}{ Overall, $n=362$} \\
\hline & $n(\%)$ & $95 \% \mathrm{CI}$ & $n(\%)$ & $95 \% \mathrm{CI}$ & $n(\%)$ & $95 \% \mathrm{CI}$ \\
\hline \multicolumn{7}{|l|}{ Prior treatment history } \\
\hline Treatment-naïve & $2(100.0)$ & $15.81-100.00$ & $286(99.7)$ & $98.07-99.99$ & $288(99.7)$ & $98.09-99.99$ \\
\hline Treatment-experienced & $1(100.0)$ & $2.50-100.00$ & $72(100.0)$ & $95.01-100.00$ & $73(100.0)$ & $95.07-100.00$ \\
\hline \multicolumn{7}{|l|}{ Sex } \\
\hline Male & $2(100.0)$ & $15.81-100.00$ & $142(100.0)$ & $97.44-100.00$ & $144(100.0)$ & $97.47-100.00$ \\
\hline Female & $1(100.0)$ & $2.50-100.00$ & $216(99.5)$ & $97.46-99.99$ & $217(99.5)$ & $97.47-99.99$ \\
\hline \multicolumn{7}{|l|}{ Age } \\
\hline$<65$ years & $3(100.0)$ & $29.24-100.00$ & 327 (99.7) & $98.31-99.99$ & $330(99.7)$ & $98.33-99.99$ \\
\hline$\geq 65$ years & 0 & NA & $31(100.0)$ & $88.78-100.00$ & $31(100.0)$ & $88.78-100.00$ \\
\hline \multicolumn{7}{|l|}{ Baseline BMI, $\mathbf{k g} / \mathrm{m}^{2}$} \\
\hline$<30 \mathrm{~kg} / \mathrm{m}^{2}$ & $3(100.0)$ & $29.24-100.00$ & $349(99.7)$ & $98.42-99.99$ & 352 (99.7) & $98.43-99.99$ \\
\hline$\geq 30 \mathrm{~kg} / \mathrm{m}^{2}$ & 0 & NA & $9(100.0)$ & $66.37-100.00$ & $9(100.0)$ & $66.37-100.00$ \\
\hline \multicolumn{7}{|l|}{ Baseline HCV RNA } \\
\hline$<800,000 \mathrm{IU} / \mathrm{mL}$ & 0 & NA & $98(100.0)$ & $96.31-100.00$ & $98(100.0)$ & $96.31-100.00$ \\
\hline$\geq 800,000 \mathrm{IU} / \mathrm{mL}$ & $3(100.0)$ & $29.24-100.00$ & $260(99.6)$ & $97.88-99.99$ & $263(99.6)$ & $97.91-99.99$ \\
\hline \multicolumn{7}{|l|}{ Baseline ALT } \\
\hline$\leq 1.5$ ULN & $3(100.0)$ & $29.24-100.00$ & $241(99.6)$ & $97.72-99.99$ & $244(99.6)$ & $97.75-99.99$ \\
\hline$>1.5$ ULN & 0 & NA & $117(100.0)$ & $96.90-100.00$ & $117(100.0)$ & $96.90-100.00$ \\
\hline
\end{tabular}

Abbreviations: ALT, alanine aminotransferase; CI, confidence interval; BMI, body mass index; ULN, upper limit of normal.

Table 3. SVR12 in the genotype 1 a subjects was $100 \%(3 / 3$, $95 \%$ CI $29.24-100.00 \%)$, and in genotype 1 b subjects SVR12 was $99.7 \%$ (358/359, 95\% CI 98.46-99.99\%).

\section{Effect of HCV NS5A and NS5B RASs on SVR12}

This study analyzed the influence of baseline mutations at amino acid sites $24,28,30,31,32,58,92,93$ in HCV NS5A and at amino acid sites $61,62,159,202,282,316,320,321$, 440 in HCV NS5B on SVR12. SVR12 in patients with NS5A RASs at baseline was $99.2 \%(130 / 131)$ and $100 \%(231 / 231)$ in patients without NS5A RAS. SVR12 in patients with NS5B RASs at baseline was $99.7 \%(355 / 356)$ and $100 \%(6 / 6)$ in patients without NS5B RAS. It was found that despite the presence of NS5A RASs or NS5B RASs at baseline, there was no effect on the treatment efficacy on SVR12.

The genotypes of the 2 subjects who failed treatment were all genotype $1 \mathrm{~b}$. One subject had no NS5A RASs but NS5B RASs (site 316) at baseline, then NS5A RASs (site 28) and NS5B RASs (site 316) at the time of relapse. Another subject had NS5A RASs (sites 28, 31, 93) and NS5B RASs (site 316) at baseline, then NS5A RASs (sites 28, 31, 93) and NS5B RASs (sites 282, 316) at the time of relapse. In 2 subjects with treatment failure, the main NS5A RASs were L28M, L31V and $\mathrm{Y} 93 \mathrm{H}$, and the main NS5B RASs were S282T and C316N. RASs in L28M and C316N were detected in both 2 subjects.

\section{Safety}

A total of 279 subjects (77.1\%) experienced AEs during the trial (Table 4). The main AEs reported for $\geq 5 \%$ of patients were upper respiratory tract infection, hypercholesterolemia, hypertriglyceridemia, hyperuricemia, and elevated serum creatine phosphokinase. One hundred and twenty-nine subjects (35.6\%) had drug-related AEs. The highest incidence of drug-related AEs was found to involve metabolic and nutritional diseases (18.2\%), followed by those detected through various examinations (17.7\%) and involving gastrointestinal diseases (5.2\%), kidney and urinary diseases $(2.5 \%)$, general disorders and administration site conditions $(2.5 \%)$, and diseases of the skin and subcutaneous tissues $(2.2 \%)$. The main treatment drugs-related symptoms reported for $\geq 2 \%$ of patients were hypercholesterolemia, hypertriglyceridemia, hyperuricemia, elevated serum creatine phosphokinase, elevated lipase, elevated serum lactate dehydrogenase, and elevated amylase.

Thirty SAEs occurred in 16 subjects, including all kinds of injuries, poisoning and surgical complications (contusion, ligament rupture, hand fracture and so on), nervous system diseases (thalamus hemorrhage, ruptured cerebral aneurysm and cerebral infarction), lung infection, appendicitis, dermal sinus, skin mass, thyroid papillary carcinoma, histiocytic necrotizing lymphadenitis, fetal abnormalities, bronchiectasis, and sudden hearing loss. All SAEs were assessed as independent of the study treatment. Most AEs were mild or moderate in severity, and did not require therapy and resolved by themselves (Table 4 ). No AEs lead to death, and no AEs lead to the premature discontinuation or interruption of the study drug.

\section{Discussion}

In this multicenter, single arm, open-label, phase 3 study, the rates of SVR12 were $99.7 \%$. This trial showed that a 12 -week 
Table 4. Adverse events frequency and severity

\begin{tabular}{|c|c|}
\hline AEs, $n(\%)$ & $\begin{array}{l}\text { Overall, } \\
n=362\end{array}$ \\
\hline Any AEs & $279(77.1)$ \\
\hline \multicolumn{2}{|l|}{ AEs reported for $\geq 5 \%$ of patients } \\
\hline Upper respiratory tract infection & $51(14.1)$ \\
\hline Hypercholesterolemia & $49(13.5)$ \\
\hline Hypertriglyceridemia & $35(9.7)$ \\
\hline Hyperuricemia & $22(6.1)$ \\
\hline Elevated serum creatine phosphokinase & $19(5.2)$ \\
\hline Treatment drugs-related AEs & $129(35.6)$ \\
\hline Grade 1 & $99(27.3)$ \\
\hline Grade 2 & $22(6.1)$ \\
\hline Grade 3 & $6(1.7)$ \\
\hline Grade 4 & $2(0.6)$ \\
\hline Grade 5 & 0 \\
\hline \multicolumn{2}{|c|}{$\begin{array}{l}\text { Treatment drugs-related AEs reported for } \geq 2 \% \text { of } \\
\text { patients }\end{array}$} \\
\hline Hypercholesterolemia & $30(8.3)$ \\
\hline Hypertriglyceridemia & $19(5.2)$ \\
\hline Hyperuricemia & $17(4.7)$ \\
\hline Elevated serum creatine phosphokinase & $14(3.9)$ \\
\hline Elevated lipase & $12(3.3)$ \\
\hline Elevated serum lactate dehydrogenase & $10(2.8)$ \\
\hline Elevated amylase & $8(2.2)$ \\
\hline SAEs & $16(4.4)$ \\
\hline $\begin{array}{l}\text { AEs leading to premature } \\
\text { discontinuation of study drug }\end{array}$ & 0 \\
\hline $\begin{array}{l}\text { AEs leading to interruption of study } \\
\text { drug }\end{array}$ & 0 \\
\hline Death & 0 \\
\hline
\end{tabular}

Abbreviations: AEs, adverse events; SAEs, serious adverse events.

regimen of emitasvir-sofosbuvir, without ribavirin, constitutes a very effective treatment for HCV genotype 1 infection patients without cirrhosis. The response to interferon-based regimens for $\mathrm{HCV}$ infection with genotype 1 was lower than other genotypes; ${ }^{5}$ however, genotype 1 patients treated with DAAs can achieve very high rates of SVR, as the SVR12 rate in this study was as high as $99.7 \%$. In the era of DAAs, genotype 1 patients became easily curable. Gower et al. ${ }^{11}$ analyzed 2320 studies on the prevalence of HCV genotypes in PubMed and EMBASE databases, including epidemiological data from 98 countries. The analysis showed that genotype 1 was the dominant genotype (in $46 \%$ of HCV infections), followed by genotype $3(22 \%)$, type $2(13 \%)$, type 4 $(13 \%)$, type $6(2 \%)$, and type $5(1 \%)$. Genotype $1 \mathrm{~b}$ is the most common subtype, accounting for $22 \%$, but there are significant national and regional variations in distribution. In China, genotype 1 accounted for 58.4\%, wherein genotype 1b accounted for $56.8 \%$ and genotype 1 a was very rare, accounting for only $1.4 \%$. Because genotype 1 a was very rare in China, only 3 patients with genotype 1 a were included in this study but all obtained SVR12. This 12-week regimen of emitasvir-sofosbuvir for genotype $1 \mathrm{~b}$ was suitable for more than $20 \%$ hepatitis C patients globally and more than onehalf hepatitis $C$ patients in China.

The response to an interferon-based regimen for HCV infection varies greatly based on certain characteristics of the patient, such as baseline viral load, ethnicity, HCV genotype, IL28B genotype, and degree of fibrosis, and whether there is an early response during treatment. ${ }^{12}$ In the era of antiviral therapy with DAAs, except for individual DAAs, baseline viral load or baseline RASs can affect efficacy, the effectiveness of many DAAs treatments is not affected by the baseline characteristics of the patient. ${ }^{13,14}$ In this trial, response rates were generally consistent, regardless of patient characteristics at baseline. For all genotype 1 patients without cirrhosis, the rates of response to a 12-week regimen of emitasvir-sofosbuvir is the same (high), regardless of baseline prior treatment history, sex, age, baseline BMI, baseline HCV RNA level, and baseline ALT level.

This study showed that HCV genotype 1 patients already had some RASs at baseline, such as R30Q and $\mathrm{Y} 93 \mathrm{H}$ in the NS5A region, C316N, E440G, and L159F in the NS5B region. The study from Wei Let al. ${ }^{15}$ showed that in HCV genotype $1 \mathrm{~b}$ patients, the prevalence of NS3 RASs was $22 \%$ in China, lower than in North America (28\%) and in Europe (40\%). The prevalence of NS5A RASs (including $\mathrm{Y} 93 \mathrm{H}$ ) was $18-21 \%$ in Asian countries, similar in North America (15\%) and Europe (19\%). The prevalence of NS5B RASs (including L159F) was $1-5 \%$ in Asian countries, lower than in North America (4\%) or Europe (20\%). RASs can reduce the efficacy of some DAA regimens, such as daclatasvir plus asunaprevir. SVR12 was lower among patients with NS5A RASs (at L31 or $\mathrm{Y} 93 \mathrm{H}$ ) than those without NS5A RASs at baseline (53\% vs. $96 \%) .{ }^{16}$ However, there is no standard kit available to detect $\mathrm{HCV}$ resistance to approved drugs. Resistance detection mainly relies on in-house techniques based on Sanger sequencing or deep sequencing. ${ }^{17}$ In our study, only 2 of the 362 patients showed virologic failure, both of whom were genotype $1 \mathrm{~b}$ patients, one with C316N RASs and the other with L28M and C316N RASs at baseline. However, the results showed that even with RASs at baseline, there was no effect on the efficacy of emitasvir-sofosbuvir. Therefore, RASs detection at baseline is not required for the treatment of genotype 1 patients with emitasvir-sofosbuvir. Virologic failure was very rare in this study's subjects, occurring in only $0.55 \%$ of patients ( 2 of 362 ); we did not identify clinical or virologic predictors of treatment failure in these 2 patients. Because both patients had RASs to NS5A inhibitors or NS5B inhibitors at baseline or at the time of relapse, a sofosbuvirbased regimen with a NS5A inhibitor and a protease inhibitor would be an option for retreatment of these 2 patients.

The most common adverse events $(\geq 5 \%)$ included upper respiratory tract infection, hypercholesterolemia, hypertriglyceridemia, hyperuricemia, and elevated serum creatine phosphokinase. Most AEs did not require special treatment and were self-limiting or self-resolving. In this trial, no AEs resulted in premature discontinuation or interruption of the study drug, and no deaths occurred. No occurrence of SAEs was related to the study drugs. The emitasvir-sofosbuvir regimen was safe and well tolerated in our Chinese patient population. This treatment regimen does not require a combination with ribavirin, which has significant blood toxicity. 
Several DAAs have been approved in China, including pangenotypic DAAs with high efficacy, such as glecaprevir/ pibrentasvir and sofosbuvir/velpatasvir. This combo of domestic NS5A inhibitors-emitasvir and sofosbuvir is expected to be less costly than the regimens that have been approved in China. It would be available to the patients with genotype $1 b$, which accounts for more than half of hepatitis $C$ patients in China, and also including those who have failed a previous interferon-based regimen.

In conclusion, our study showed that the 12 weeks of treatment with emitasvir-sofosbuvir was a highly efficient and safe treatment for a wide range of patients with $\mathrm{HCV}$ genotype $1 \mathrm{~b}$ infection without cirrhosis, who had either not been treated or who had been previously treated with interferon-based regimen. This regimen would be ideal for populations where genotype $1 \mathrm{~b}$ is predominant such as northern, central and eastern China, and Europe.

\section{Funding}

This work was supported by the National Major Scientific and Technological Special Project for "Significant New Drugs Development" during the Thirteenth Five-Year Plan Period of China (Nos. 2017ZX09201006004 and 2017ZX09201006009), the Chinese National Research Grant of the Thirteenth Five-Year Plan for the Key Projects in Infectious Diseases (No. 2017ZX10202202), the Key Research and Development Program of Guangdong (No. 2019B02021002), and was funded by Sunshine Lake Pharma Co., Ltd. (ClinicalTrials.gov number, NCT 03487107).

\section{Conflict of interest}

Huiying Rao has received speaking fees from Bristol-Myers Squibb, Gilead, and AbbVie. Hongming Xie, Lin Luo, Qingyun Ren, and Yingjun Zhang are employees of Sunshine Lake Pharma Co., Ltd. Lai Wei has received research support and/ or consulting fees from Bristol-Myers Squibb, Roche, and Novartis. The other authors have no conflict of interests related to this publication.

\section{Author contributions}

Conception and design (HYR, YJZ, LW), data collection (HYR, XXY, YWT, QN, DY, JFW, YFY, SZ, DLY, JLH, QX, CYZ, LLZ, XRM, TS, LB, FCZ, JLJ, YGZ, MRW, WX, YJM, JQ, ZBY, PA, FL, JDJ, $X X H, Z J G, J W, Y P C, Z S J, M H L, G Q W, Y Y Z, R H)$, data analysis and interpretation (HYR, HMX, LL, QYR, YJZ, LW), manuscript writing (HYR, LW).

\section{References}

[1] World Health Organization. Guidelines for the care and treatment of persons diagnosed with chronic hepatitis $C$ virus infection. Available from: https: //www.who.int/hepatitis/publications/hepatitis-c-guidelines-2018/en/

[2] She LH, Lin CS, Cai QX, Zhang XH, Wu YK, Zhao ZX, et al. A five-year followup of one hundred and thirty-six patients of hepatitis C. Zhonghua Gan Zang Bing Za Zhi 2011;19:823-827. doi: 10.3760/cma.j.issn.1007-3418.2011. 11.008.

[3] Rao H, Wei L, Lopez-Talavera JC, Shang J, Chen H, Li J, et al. Distribution and clinical correlates of viral and host genotypes in Chinese patients with chronic hepatitis C virus infection. J Gastroenterol Hepatol 2014;29:545-553. doi: 10.1111/jgh.12398.

[4] Chen Y, Yu C, Yin X, Guo X, Wu S, Hou J. Hepatitis C virus genotypes and subtypes circulating in Mainland China. Emerg Microbes Infect 2017;6:e95. doi: $10.1038 / \mathrm{emi} .2017 .77$.

[5] The guideline of prevention and treatment for hepatitis C: a 2015 update. Zhonghua Gan Zang Bing Za Zhi 2015;23:906-923. doi: 10.3760/cma.j. issn.1007-3418.2015.12.003.

[6] Wei L, Lim SG, Xie Q, Văn KN, Piratvisuth T, Huang $Y$, et al. Sofosbuvirvelpatasvir for treatment of chronic hepatitis $C$ virus infection in Asia: a single-arm, open-label, phase 3 trial. Lancet Gastroenterol Hepatol 2019; 4:127-134. doi: 10.1016/S2468-1253(18)30343-1.

[7] Wei L, Jia JD, Wang FS, Niu JQ, Zhao XM, Mu S, et al. Efficacy and safety of elbasvir/grazoprevir in participants with hepatitis $C$ virus genotype 1, 4, or 6 infection from the Asia-Pacific region and Russia: Final results from the randomized C-CORAL study. J Gastroenterol Hepatol 2019;34:12-21. doi: 10.1111 /jgh.14509.

[8] Wei L, Xie Q, Hou JL, Tang H, Ning Q, Cheng J, et al. Ledipasvir/sofosbuvir for treatment-naive and treatment-experienced Chinese patients with genotype $1 \mathrm{HCV}$ : an open-label, phase 3b study. Hepatol Int 2018;12:126-132. doi: 10.1007/s12072-018-9856-z.

[9] Guidelines for the prevention and treatment of hepatitis C (2019 version). Zhonghua Gan Zang Bing Za Zhi 2019;27:962-979. doi: 10.3760/cma.j. issn.1007-3418.2019.12.008

[10] Luo BF, Jing JL, Rao HY, Ning Q, Hou JL, Bai L, et al. Efficacy and safety of yimitasvir phospha combined with sofosbuvir in patients with chronic hepatitis C virus infection. Chin J Infect Dis 2019;37:420-429. doi: 10.3760/cma. j.issn.1000-6680.2019.07.007.

[11] Gower E, Estes C, Blach S, Razavi-Shearer K, Razavi H. Global epidemiology and genotype distribution of the hepatitis $C$ virus infection. J Hepatol 2014; 61:S45-S57. doi: 10.1016/j.jhep.2014.07.027.

[12] Ghany MG, Nelson DR, Strader DB, Thomas DL, Seeff LB. An update on treatment of genotype 1 chronic hepatitis $C$ virus infection: 2011 practice guideline by the American Association for the Study of Liver Diseases. Hepatology 2011;54:1433-1444. doi: 10.1002/hep.24641.

[13] EASL Recommendations on Treatment of Hepatitis C 2018. J Hepatol 2018; 69:461-511. doi: 10.1016/j.jhep.2018.03.026.

[14] AASLD-IDSA HCV Guidance Panel. Hepatitis C guidance 2018 update: AASLD-IDSA recommendations for testing, managing, and treating hepatitis C virus infection. Clin Infect Dis 2018;67:1477-1492. doi: 10. 1093/cid/ciy585.

[15] Wei L, Omata M, Lim YS, Xie Q, Hou JL, Jia J, et al. HCV phylogenetic signature and prevalence of pretreatment NS5A and NS5B NI-Resistance associated substitutions in HCV-Infected patients in Mainland China. Antiviral Res 2018;158:178-184. doi: 10.1016/j.antiviral.2018.08.001.

[16] Wei L, Wang FS, Zhang MX, Jia JD, Yakovlev AA, Xie W, et al. Daclatasvir plus asunaprevir in treatment-naïve patients with hepatitis $C$ virus genotype $1 \mathrm{~b}$ infection. World J Gastroenterol 2018;24:1361-1372. doi: 10.3748/wjg. v24.i12.1361.

[17] Fourati S, Pawlotsky JM. Virologic tools for HCV drug resistance testing. Viruses 2015;7:6346-6359. doi: 10.3390/v7122941. 